\title{
Migraine generator network and spreading depression dynamics as neuromodulation targets in episodic migraine
}

\author{
Markus A. Dahlem 1 ,a) \\ Institute of Physics, Humboldt-Universität zu Berlin, Berlin, Germany
}

(Dated: 16 May 2013)

\begin{abstract}
Migraine is a common disabling headache disorder characterized by recurrent episodes sometimes preceded or accompanied by focal neurological symptoms called aura. The relation between two subtypes, migraine without aura (MWoA) and migraine with aura (MWA), is explored with the aim to identify targets for neuromodulation techniques. To this end, a dynamically regulated control system is schematically reduced to a network of the trigeminal nerve, which innervates the cranial circulation, an associated descending modulatory network of brainstem nuclei, and parasympathetic vasomotor efferents. This extends the idea of a migraine generator region in the brainstem to a larger network and is still simple and explicit enough to open up possibilities for mathematical modeling in the future. In this study, it is suggested that the migraine generator network (MGN) is driven and may therefore respond differently to different spatio-temporal noxious input in the migraine subtypes MWA and MWoA. The noxious input is caused by a cortical perturbation of homeostasis, known as spreading depression (SD). The MGN might even trigger SD in the first place by a failure in vasomotor control. As a consequence, migraine is considered as an inherently dynamical disease to which a linear course from upstream to downstream events would not do justice. Minimally invasive and noninvasive neuromodulation techniques are briefly reviewed and their rational is discussed in the context of the proposed mechanism.
\end{abstract}

Migraine is characterized by recurrent attacks of moderate to severe headaches sometimes preceded by visual, sensory, motor, or language disturbances. These so-called migraine aura symptoms are caused by a chemical imbalance in the brain that lasts usually not longer than one hour, while the longer lasting headaches originate from neural activity in the brainstem. Both have been observed with non-invasive imaging. In this study, it is proposed how these events are linked together and that migraine pathophysiology should be considered as a dynamical network phenomenon.

\section{INTRODUCTION}

Migraine is one of the most prevalent neurological disorders with a life-time prevalence of about $14 \%$. Migraines can cause substantial levels of disability, ranking on a disability scale from $0.0-1.0$ at $0.7^{1}$. A migraine attack can have up to four distinct stages: a prodromal phase with difficulty concentrating, yawning, and fatigue; an aura phase with all kinds of sensory disturbances; the headache phase, often unilateral and throbbing; and finally a postdrome of tiredness, difficulty concentrating, persistence of sensitivity to light and noise. Yet, despite a distinctive clinical picture, migraine continues to be underdiagnosed and undertreated. It is estimated that the cost for the US and European economies sum up to US $\$ 19.6$ billion and $€ 27$ billion a year, respectively.
There are several subforms of migraine, yet the two main subtypes are migraine without aura (MWoA) and migraine with aura (MWA). MWA comprises, for instance, six subforms, one even without headache. While for most migraine sufferers, the head pain is the key manifestation of their disorder, the migraine aura is also a distinctive feature: often visual hallucinations, language problems, motor weakness and other short-duration (5$60 \mathrm{~min}$ ) changes to sensory modalities or cognitive functions. These symptoms occur only in MWA, i.e., in about $30 \%$ of the cases and usually precede or sometimes accompany the headache ${ }^{2}$. Why not in all attacks? This raises a related more general question: Is the pain mechanism in MWoA and MWA the same? If so, what drives the activation of neuronal pain pathways and how is this related to the cause of aura symptoms? And what determines which sensory modality or cognitive function is affected? These interlinked questions about migraine pathophysiology remain open or are only unsatisfactorily answered in current migraine theories.

The migraine generator and the spreading depression (SD) theory of migraine both focus on distinct pathophysiologic events, yet they are not mutually exclusive theories of the cause of episodic migraine. The issues raised above might be resolved, if both theories can be unified.

From a mathematical point of view, migraine pathophysiology involves sudden dynamical transitions, because although migraine is a chronic neurological disorder it is characterized by recurrent episodes. In these transitions both temporal rhythms and spatio-temporal patterns change, which allows only one conclusion: migraine is a dynamical disease; a term coined to identify diseases that occur due to an abrupt change, usually a

\footnotetext{
a) dahlem@physik.hu-berlin.de: https://sites.google.com/site/markusadqblfqmetation. In fact originally, dynamical diseases are de-
} 
fined as a situation where sudden changes bring the system through a bifurcation ${ }^{3}$. But in a wider sense, if we have - or aim for - an understanding of the pathophysiological mechanism in terms of equations of motion and low dimensional phase space structures, we may call it a dynamical disease. The sudden change may also originate from other dynamical phenomena than bifurcations, such as intermittency, noise driven excitation behavior, etc.. These various points that lead to sudden transitions may also be summarized as "tipping points".

As a consequence, migraine pathophysiology needs to be considered as a problem at the interface of clinical neurology and applied nonlinear science. To this end, the migraine generator theory must be extended from a theory of brainstem regions as the origin of attacks to a network concept where the origin is not localized but a dynamical transition into dysfunctional contro ${ }^{4 / 7}$. In Sec. II], an extended but still reduced migraine generator network (MGN) will be introduced. This network is connected to the cortex, where the spatio-temporal patterns of SD occur. SD itself can be describes by a macroscopic continuous limit of cortical tissue in terms of reaction-diffusion systems (Sec. III). How do these two dynamical systems interact in migraine, that is, how is the central pattern generator network coupled to the cortical reaction-diffusion system?

A possible relation is proposed in this theoretical study. The pivotal role lies in the driving input of the MGN. The MGN is a system that compromises two physiological control subsystems, the trigeminovascular system for vasomotor control and associated descending modulatory brainstem system for pain control. The vasomotor system may even lead to the ignition of cortical SD in the first place, which then in turn drives the MGN. We do not consider the cause of the ignition of SD but suggest SD - once ignited - drives the MGN and this systems responds differently to different spatio-temporal signatures of SD, that is, characteristic noxious action of SD leads to the subforms MWA and MWoA. In this study, only the driving mechanism is considered in some detail in Sec. [III.

A mathematical model of this dynamical response is beyond the scope here, though the MGN itself is presented as a reduced scheme explicit enough to support the conclusions for neuromodulation techniques (Sec. IV). In general, this dynamical response is a form of central sensitization, which refers to a transition in a pain networkpart of the central nervous system - after which it responds with increased sensitivity to noxious stimuli (hyperalgesia) and even non-noxious stimuli (allodynia). In episodic migraine, the central sensitization is probably one of second-order neurons ${ }^{8}$. Chronic migraine, in contrast, central sensitization is probably a dynamical transition in the pain matrix (see below), which also leads to an enhanced response to peripheral input but is not considered here.

In Sec. IV] minimally invasive and noninvasive neuromodulation techniques are briefly reviewed. These tech-
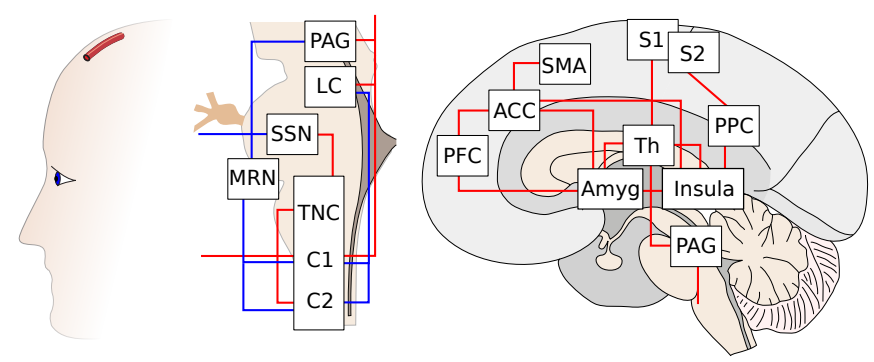

FIG. 1. (color online) Networks related to pain. (a) Cranial circulation (CC): connected by first-order perivascular pain-sensitive neurons via the trigenimal ganglion (TG) to the trigeminocervical complex (TCC, see b) and receives also input from the superior salivatory nucleus (SSN). (b) Brainstem: trigeminal ganglion (TG); trigeminal nucleus caudalis (TNC) and C1-C2 regions compromise the trigeminocervical complex (TCC); rostral ventromedial medulla (RVM), locus coeruleus (LC), ventrolateral periaqueductal grey (vlPAG). (c) Pain matrix ${ }^{62}$; thalamus (TH); anterior cingulate cortex (ACC); amygdala (Amyg); (PFC); primary (S1) and secondary (S2) somatosensory cortices; supplementary motor area (SMA); prefrontal cortex (PPC); insula cortex (Insula).

niques target neural structures in the peripheral and central nervous system that have been introduced in Sec. II and Sec. III. The aim of these techniques is to abort migraine. I discuss their rational in the context of the proposed driving mechanism of the MGN by SD dynamics.

In conclusion, noninvasive techniques for episodic migraine should be most effective in transcranial stimulation with three distinct modes of action, for (i) the prodromal phase, (ii) the ignition and (iii) the acute phase of migraine aura. Furthermore, one mode of action in transcutaneous stimulation (iv) in the acute pain phase of the episodic attacks. Further quantitative approaches are needed to find optimized stimulation protocols for these situations based on the proposed mechanism.

\section{MIGRAINE GENERATOR NETWORK}

In several respects, pain is different from other sensations. For instance, normal sensory pathways take a chain of three neurons to get from receptors, e.g., sensitive mechanoreceptors, to the primary sensory cortical area. In pain traffic, there are more intermediate nuclei and a cascade of descending control mechanisms is essential for defining the pain experience ${ }^{9}$. Therefore a simple order of neurons on the way to the cortex is questionable. Furthermore, instead of a single, i. e., spatially confined, primary cortical area as the first target in the cortex, a neural correlate of pain perception seems to be found in spatially segregated activity patterns of a widespread network of cortical areas.

Whether there is a network specific for pain perception, the "pain matrix" — a term that usually also in- 
cludes diencephalic structures in the forebrain such as the thalamus, see Fig. 1 or not and instead this network actually is a largely unspecific sensory "neuromatrix", is still debated 10 . In any case, this matrix seems not to be an optimal target in episodic migraine treatment, e.g., for neuromodulation techniques, because its activity patterns are probably truly downstream events (but cf. chronification of pain, i. e., $>15$ headache days per month over a 3 month period, at least 8 migrainous, and absence of medication overuse).

The question of the most upstream event is more difficult (see Discussion), and it may only make sense to talk about upstream events in the form of transitions in dynamical states of the migraine generator and spreading depression (Sec. III).

While it is worth illustrating all nuclei of this network in their respective anatomical place (in approximation), it is still necessary to identify a schematically reduced network of these nuclei, their neural subpopulations, and a vasomotor loop that is explicit enough to convey the regulatory control in this network structure amenable for mathematical analysis, see Fig. 2 For this reason, the following brain regions and their respective functions are briefly introduced.

\section{A. The trigeminovascular system}

The trigeminovascular system, which is key to understanding the network structure of the MGN, is a largely bidirectional system. Notwithstanding, it is natural to consider as a starting point, if only for convenience, the nociceptor and the afferent projection of the first-order neuron, that is, the innervation of both dura mater and large intracranial vessels by the ophthalmic division of the trigeminal nerve. This innervation of the cranial circulation (CC) terminates in the central trigeminal nucleus caudalis (TNC) and the $\mathrm{C} 1-\mathrm{C} 2$ regions of the cervi-

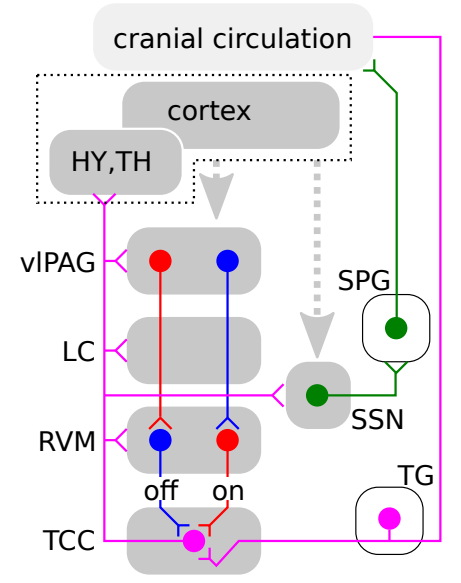

FIG. 2. (color online) Schematic diagram of pathways in the migraine generator network (MGN) cal spinal cord, together known as the trigeminocervical complex (TCC), see Fig. 1 .

Beyond the TCC, nociceptive traffic ascends by activated second-order neurons to further brainstem nuclei: the rostral ventromedial medulla (RVM) including the nucleus raphe magnus (NRM) in the medulla; the locus coeruleus (LC) in the dorsolateral pons (both hindbrain); and the ventrolateral periaqueductal grey (vlPAG) in the tegmentum (midbrain). Furthermore, the TCC also ascends nociceptive information directly to the hypothalamus (HY) and thalamus (TH) with secondorder neurons along the trigeminohypothalamic tract and the trigeminothalamic tract (also know as quintothalamic tract), respectively. $\mathrm{TH}$ and $\mathrm{HY}$ are structures in the diencephalon of the forebrain from where third-order neurons ascend to the cortex (CTX), the final telencephalic station within the forebrain.

For the sake of completeness, one other pathway of the trigeminovascular systems should be mentioned. There is a peripheral vasomotor connection from efferents of the superior cervical spinal cord to the cranial circulation 11 .

\section{B. Cortical and diencephalic influence}

Before we come to the descending modulatory network of the brainstem, let us briefly mention descending pathways from the pain matrix. Cortical projections to the periaqueductal grey (PAG) arise from the medial network of the prefrontal cortex (PFC) or cortical areas that are closely related to this network $\frac{12}{12}$. The interest here is obviously the part which belongs to the MGN, in particular the ventrolateral (vl) PAG. The vlPAG evokes passive coping reactions (withdrawal, quiescence ${ }^{13}$ consistent with behavior exhibited by migraine patients. Since such coping behavior is also readily elicited in animals with the forebrain being disconnected from the brain stem $[13$, a less important role of these descending pathways for the dynamics of the MGN is expected.

Therefore, we do not include the detailed areas or even a closed feedback loop through the pain matrix in the attempt to construct a basic structure of the MGN in Fig. 2, though this input is indicated by a dotted grey arrow. To support this further, it could be shown with non-invasive imaging that brainstem activation in humans persists in migraine even after pain relief and reduction of symptoms by acute treatment ${ }^{4}$. However, it might be that not PAG but nearby nuclei in the dorsolateral pons are causing the activation ${ }^{7}$.

Notwithstanding, the thalamus is certainly key for integration of nociceptive inputs in migraine and the pain matrix for pain sensation 14 . In particular to understand chronification of pain, closed feedback loops through these areas are probably important and these diencephalic or cortical areas may even primarily be the "upstream" target areas for a therapeutic rational in chronic migraine. 


\section{Descending modulatory network}

Given that PAG is an area to which various cortical and diencephalic structures project, it is important to note that PAG does not directly project itself to the TCC. The descending projection from PAG modulate ON and OFF neurons in RVM. As indicated by the name, these $\mathrm{ON}$ and $\mathrm{OFF}$ neurons in the PAG-RVM descending control system can be facilitatory and inhibitory depending on how the balance shifts in either direction 15 . A descending facilitation of TCC can provide a mechanism for central sensitisation. The development of cutaneous allodynia suggests that the balance shifts in favor of facilitation explaining the increased sensitivity of the skin to non-noxious stimuli in migraine ${ }^{16}$.

It has been questioned whether the PAG or rather the nuclei in the dorsolateral pons, like LC, are responsible in this descending modulatory network and it was suggested that "[t]o advance the brainstem migraine generator theory from the opinion phase to being evidence based, answers should be provided to questions as: (a) How would increased activation in the PAG drive or produce migraine?"7. Quantitative approaches in a form of a mathematical model similar to the gate theory of firstorder neurons ${ }^{17}$ may contribute to this question. The important notion stressed here is that the extended migraine generator network theory is essentially a dynamic network theory. The MGN is a central pattern generator, that is, a neural network that produces rhythmic patterned output perceived as pain without sensory feedback. However, such feedback is still needed, as proposed here, to drive the transition into central sensitization. The explanatory power of a mathematical approach lies in the predictive power, but it certainly also needs the clinical testing to be evidence based.

\section{Vasomotor control}

The key vasomotor control is probably played by a parasympathetic cluster of neuronal cell bodies located in a fossa (ditch) in the skull, the sphenopalatine ganglion (SPG). The simplest putative structure of the MGN (Fig. 2) is completed by the SPG and its secondorder preganglionic neurons in the superior salivatory nucleus (SSN). It was suggested that migraine triggers typical for the prodromal phase either activate or even originate in a number of brain areas whose projections converge on the SSN (dotted grey arrow) and which are functionally positioned to produce migraine symptoms 8 .

\section{SPREADING DEPRESSION THEORY OF MIGRAINE}

SD is essentially a slow (about $3 \mathrm{~mm} / \mathrm{min}=50 \mu \mathrm{m} / \mathrm{sec}$ ) reaction-diffusion wave in gray matter tissue. In the cor- tex, SD is accompanied by a pronounced hemodynamic response of increased regional cerebral blood flow (hypermia) for about $2 \mathrm{~min}$ and a long lasting, $\sim 2 \mathrm{~h}$, decrease (oligemia) $)^{18}$.

The cortical tissue SD traverses is massively perturbed in its ion homeostasis 19 . If the ion flow across the membrane in this region is taken to estimate relative changes in Gibbs free energy of the tissue, SD reveals itself as "a twilight state close to death" 20, i. e., during the peak of SD the cortical state is similar to the one in stroke or ischemia. The ion flow causing this state is far more dramatic than in any other neurological disorders, for instance, compared to the ion flow during ictal epileptic activity.

However, the cortex is not pain sensitive. It was therefore suggested that SD may trigger the pain phase indirectly 2122.

\section{A. SD and pain pathways}

In several animal models, possible pathways were investigated to see how SD might cause pain by activating nociceptors in sensitive intracranial tissues and subsequently activate the $\mathrm{TCC}^{23}$. In short, it was suggested that during SD the blood-brain barrier becomes more permeable, allowing glutamate, potassium ions, hydrogen ions, nitric oxide and other noxious substances or inflammatory mediators to diffuse from the surface of the cortex into the meninges where they activate nociceptors of the cranial circulation. Although this mechanism has been criticized 24 , the animal models of migraine show that SD activates the first-order neurons in the trigeminal nucleus caudalis $21|22| 25 \mid 26$. In a newly published article, it was found that SD can trigger headache by activating neuronal Panx1 channels, a megachannel that is a structural component of gap junctions 27 . It is furthermore discussed, whether nuclei in the MGN in turn can cause spreading depression in the cortex. For a summary, see Ref.28.

\section{B. SD, aura, and silent courses}

Even the apparently solved question of how SD relates to the aura phase has still some merit. It is clear that the aura is caused by SD. At least after successfully imaging the blood flow in migraine aura, it became indisputable that SD is the electrophysiological correlate of migraine aura 29 . In fact, already in 1945, Leão wrote: "The latter disease [migraine] with the marked dilatation of major blood vessels and the slow march of scotomata [sensory blindness] in the visual or somatic sensory sphere is suggestively similar to the experimental phenomenon here described [SD], in spite of the fact that known scotomata are still felt to be vasoconstrictor in nature" 32 . It was not known in 1945 that in migraine, a vasoconstrictor response (oligemia) is also present. 


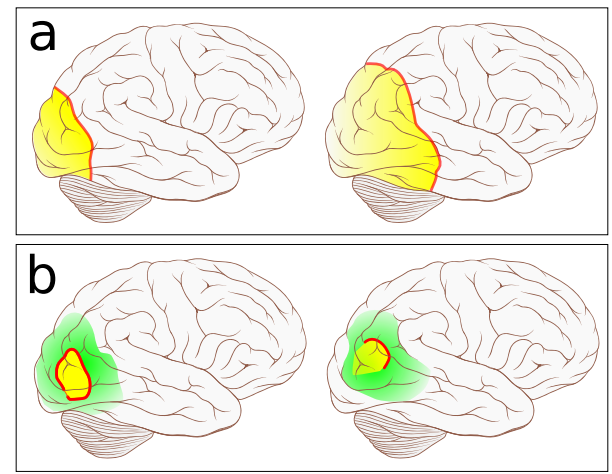

FIG. 3. (color online) Paradigms of SD pattern in migraine. Narrow band of excitation (red), refractory zone (yellow), disperse neurovascular feedback (green). (a) Typical textbook illustration: SD engulfing large parts of one hemisphere; adopted from Ref ${ }^{[30}$. (b) Localized SD wave in a full-scale $\operatorname{attack} 3136$.

To date, what remains disputed is whether SD is even present but clinically silent in the migraine subtype MWoA. This is referred to by the contested notion of "silent aura"28. If true, it is SD which stays silent, the aura is simply not present. Therefore "silent SD" is a term less perplexing. Anyway, such silent SD courses are supported by (a) a well-documented case of blood-flow changes that were likely the result of SD observed in a spontaneous migraine headache without aura ${ }^{33}$ and (b) the fact that SD can cause pain and MWA and MWoA are likely to share the same pain mechanism.

To resolve these questions about the relation of SD to the pain and aura phase, we recently suggested that which of the two migraine subtypes develops, i. e., MWoA or MWA, is dependent upon the spatio-temporal pattern (shape, size, and duration) of $\mathrm{SD}^{34}$. This does not rule out the possibility that SD itself is triggered by events in the brainstem and therefore we contribute only little to the questions of the most upstream event. However, the crucial part missed in the current controversy might be the spatio-temporal pattern of the cortical tissue being recruited into a depleted state. All too often, it is completely ignored that SD usually propagates in the human gyrified cortex as a discontinuous wave segment as observed with fMRI in migraine and reported by patients as visual field defects3135136, see Fig. 3b. In animal models, SD is engulfing the whole lissencephalic cerebral hemisphere but already in gyrencephalic cat brain only the first SD waves do this and succeeding secondary $\mathrm{SD}$ waves, which propagate in relative refractory, i.e., less susceptible, cortex, often remained within a confined $\operatorname{origin} 37$.

\section{Localized SD waves}

Both fMRI data and reports on visual field defects 5135136 clearly show that SD is not only spatially confined in humans, but SD is a discontinuous wave - a particle-like wave. A particle-like wave is a wave front with two open ends, sometimes also called dissipative soliton ${ }^{38}$. The existence of such localized waves is a change in the pattern forming paradigm indicating a fast diffusing second inhibitor related to the Turing bifurcation.

From a clinical perspective, migraine aura symptoms are focal symptoms. If SD were to spread out in all directions, the affected cortical area would increase quickly and symptoms should become more generalized. Localization does not merely restrict the symptoms caused by SD; but localization also allows SD to assume a much larger variety of spatio-temporal patterns compared to a continuous circular wave front. This is reflected in the variety of the courses of focal aura symptoms ${ }^{39}$ and maybe also in the fact that SD could stay silent under certain conditions on the localised pattern (Sec. III B,F) and, furthermore, that SD does not cause pain under other conditions (Sec. III F-G).

For these reasons, it is an essential feature that SD stays localised even when it propagates a long distance in one direction. In contrast, if SD spread out in all directions, as to date shown in all modern migraine textbooks (published or revised in the last 5 years), the shape would essentially be circular (cortical folding has some influence) and, furthermore, size and duration both would depend on a single parameter, the radial distance SD travels. The course that is usually sketched starts from the occipital pole of the cortex and SD propagates in a full-scale MWA attack up until the frontal lobe, see Fig. 3a. If SD invaded the complete occipital, temporal, and parietal lobe, this would not match with the reported courses of neurological symptoms during the aura phase, not even in a full-scale attack, cf. Ref $\frac{39}{\text {, }}$, and it is also implausible that only a subset of the nearly completely depleted cortical tissue results in some form of clinical manifestation.

A simple calculation illustrates the different scaling behavior of events. In a full-scale MWA attack, the aura phase can last up to one hour. This corresponds to a length $l$ of $l_{1}=18 \mathrm{~cm}$ cortex being paced. Even if we halve this distance $l_{2}=9 \mathrm{~cm}$ - either because SD is slower or lasting only $30 \mathrm{~min}$ and symptoms reverberate for a full $60 \mathrm{~min}$ - this would still correspond to $20 \%$ $\left(80 \%\right.$ for $\left.l_{1}=18 \mathrm{~cm}\right)$ of the surface area of one cerebral hemisphere. In contrast, only $1.5 \%$ ( $3 \%$ for $\left.l_{1}=18 \mathrm{~cm}\right)$ of the surface are affected in a full-scale MWA, if the particle-like SD wave is on average $2 \mathrm{~cm}$ wide. Note that the progressive development of aura symptoms is, except for the ignition phase of SD, probably mostly due to the fact that SD starts in regions of high cortical sensory magnification and travels into regions with lower cortical magnification 40 . 


\section{Generic reaction-diffusion model}

In accordance with the noninvasively imaged SD progression and reported visual field defects, we proposed a model in which in each attack a particular pattern is formed by a discontinuous wave segment that spreads out only in one direction determined by the initial conditions $\frac{34}{34}$ and guided by the folding pattern (unpublished results). The ignition patterns that trigger these simulated attacks are given by an assumed hyperactivity in a cortical feature map of the visual cortex used as initial conditions for the model. These feature map patterns were also suggested to be hyperactive during SD causing visual hallucinatory percepts 41 .

In this generic reaction-diffusion mechanism of excitable media, the resulting pattern forming processes following these initial conditions are described in abstract terms of activator-inhibitor kinetics of two variables $u$ and $v$, respectively. In fact, simple activator kinetics of $u$ date back to a mechanism of SD described in 1963 by Grafstein, Hodgkin and Huxley $(\mathrm{GHH})^{42}$. The GHH model assumed $u \equiv\left[K^{+}\right]_{e}$, i.e., the extracellular potassium ion concentration to be the activator $u$. We extended this scheme (for details of the full model see Ref. $\left.{ }^{34}\right)$ :

$$
\begin{aligned}
& \frac{\partial u}{\partial t}=u-\frac{1}{3} u^{3}-v+D \nabla^{2} u \\
& \frac{\partial v}{\partial t}=\varepsilon\left(u+\beta+K \int H(u) \mathrm{d} x \mathrm{~d} y\right),
\end{aligned}
$$

with $D$ the diffusion coefficient, two parameters $\varepsilon$ (time scale separation) and $\beta$ (threshold), $H$ being the Heaviside step function (discontinuous function whose value for negative (positive) arguments is 0 (1) and 0.5 for the argument zero). The integral term is therefore a surface area measure of the medium being in a state with $u$ larger than zero that can globally increase the threshold.

Both activator $u$ and inhibitor $v$ are lump variables. Unlike in the original GHH model, it is not necessary or even possible to identify particular physiological quantities, like $\left[K^{+}\right]_{e}$, with them. Instead, the activator $u$ should be viewed as the bistable energy state. One stable fixed point corresponds to the maintenance of homeostasis far from thermodynamic equilibrium and the other stable fixed point to the state where the cellular Nernst reversal potential are depleted (thermodynamic equilibrium). In the presence of an inhibitor $v$, which was not included in the GHH model, the second stable fixed point becomes a transient state. In other words, the inhibitor $v$ is a recovery variable. It is related to ion pumps that drive the tissue far from thermodynamic equilibrium and (re)charge Nernst reversal potentials. We set the parameters as follows: time scale separation $\varepsilon=0.04$, the threshold $\beta=1.32$, and the mean field coupling $K=0.003$.

The details of the model are discussed elsewhere ${ }^{34 \mid 35}$. In short, for $K=0$, the bistable activator kinetics with diffusion and the linear inhibitor kinetics (immobilised) provide the simplest reaction-diffusion model of activator-inhibitor type for the cortex as an excitable media exhibiting patterns as seen during SD in animal models. The homogeneous steady state is a stable solution representing the healthy cortical state. A critical neuronal mass is needed to start ignition and support sustained propagation of a Gibbs free energy-depleted state, which is associated with the state of $\mathrm{SD}^{20}$. For $K=0$, this depleted SD state would engulf all the tissue after an initial ignition. The suggested pattern forming mechanism of localized SD is closely related to the critical mass needed for ignition, called a critical nucleation solution, see Fig. 4a.

This critical nucleation is controlled for $K \neq 0$ by a widely spread out inhibitory feedback, Fig. $4 \mathrm{~b}, \mathrm{c}$. This inhibitory feedback is approximated by a mean field term $K \int H(u) \mathrm{d} x \mathrm{~d} y$, a term proportional to the depleted surface area region in the cortex. The physiological significance of this last term in the rate function of the lump variable $v$ is associated with the neurovascular feedback (e.g., as observed during hypermia of increased blood flow ${ }^{29131}$ ) in SD. For the sake of simplicity, we chose it to be proportional to the surface area of the depleted state, although the neurovascular feedback is mainly driven by the neuronal hyperactivity in the rise of the SD front. Therefore, a measure that is proportional to the length of the SD front may be more appropriate. However, we tested $K \int H(u) H(1-v) \mathrm{d} x \mathrm{~d} y$, which is an approximated measure of the length of the SD front - the activator must be in the excited state while the inhibitor is still low, as characteristic for the hyperactivity in the

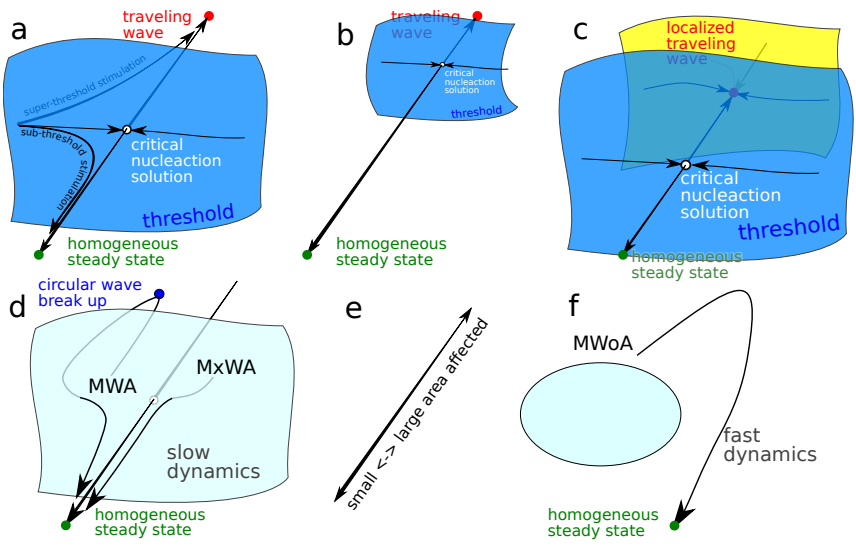

FIG. 4. (color online) Schematic orbit structures in phase space $^{34}$, see text. (a) Caricature of excitable media. (b) Basin of attraction change when local excitabilty parameter change. (c) Fast secondary inhibition creates localized structures. (d) Ghost behaviour when traveling wave solution (node) collides with nucleation (saddle): the two trajectories are associated with migraine with aura (MWA) and with migraine with typical aura without headache (MxWA). (e) Direction of cortical area affected by SD. (f) Trajectory of a circular wave associated with migraine without aura (MWoA). 
rising front-with similar results.

\section{E. Transient waves and slow dynamics}

Changing a parameter of this model without the mean field inhibitory feedback $(K=0)$, essentially varies the basins of attraction of the existing solutions, e. g., making the medium less susceptible by increasing the basin of attraction of the homogeneous steady state, Fig. 4p. For $K \neq 0$, the traveling wave solution itself is changed such that the traveling wave solution becomes localized, as described in the previous section, Fig. 4k. For sufficiently large $K$, this stable localized solution collides with its nucleation solution (saddle), which leads to well known phenomenon of slow dynamics named ghost behavior after a saddle-node bifurcation, Fig. $4 \mathrm{~d}$. This is the origin of the transient nature of the SD signatures in Fig. 5 that we propose to lead to migraine without aura, migraine with aura, and migraine with typical aura and without headache (MWoA, MWA, and MxWA, respectively).

In particular, MWA, and MxWA correspond to trajectories that pass the ghost of the slow manifold (lightblue in Fig. 4d). In contrast, solutions that are shorter lasting but with larger MIA (cf. Fig. 4e) do not pass the ghost of the slow manifold Fig. 4; they correspond to MWoA.

In Sec. IV D, this is further discussed in the context of the therapeutic strategy for neuromodulation.

The macroscopic features of SD are well described by this model. The affected cortical area will not grow in time and SD stays localized while propagating (for further discussion of the usefulness of such a toy model, see Sec. IV D . It is worth noting that it is not without irony that both terms in the name of SD are rather misleading: the important neural activity is a hyperactivity that only in the depleted phase becomes "depressed", and this cortical state is only in the ignition phase "spreading", the sustained main process is a traveling wave.

\section{F. Link to migraine subforms}

We performed statistical analysis of the spatiotemporal development governed by the generic reactiondiffusion model in Eq. (1)-(2) that followed 8000 different natural initial perturbations of local hyperactivity as SD triggers. These perturbations were introduced by an increase of the resting value of $u$. Based on that, we predict that certain features related to shape, size, and duration of SD (Fig. 5a) determine the aura phase while others determine the pain phase in migraine.

It is important for further discussion that we firstly divide the subtype MWA into its two subforms "typical aura with migraine headache (1.2.1, in the international classification)" and "typical aura without headache (1.2.3)", which we refer to as MxWA. Therefore, in the remainder of the text, we refer to 1.2.1 as MWA, i.e.,
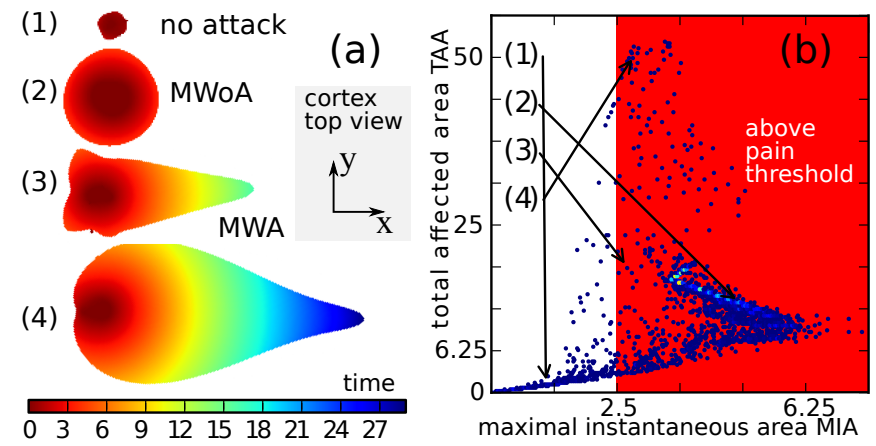

FIG. 5. (color online) (a) Spatio-temporal signatures of SD as obtained from a reaction-diffusion model with mean field coupling, Eqs. (1)-(2); cf. Fig. $4 \mathrm{E}$ in Ref. ${ }^{31}$. The model parameters are chosen such that only transient waves exist ${ }^{34}$, note that we have scaled the dimensionless model such that the units of time are roughly corresponding to minutes, and surface area to $\mathrm{cm}^{2}$ and the wave spreads with about $3 \mathrm{~mm} / \mathrm{min}$. Four stereotypical courses are depicted following a local perturbation of a homogeneous steady state: (1) subthreshold, SD dies out quickly without initial spread; (2) superthreshold perturbation, SD dies out after a few minutes, if its front does not break open, corresponding to migraine without aura (MWoA); (3)-(4) superthreshold perturbation, SD propagates for up to $30 \mathrm{~min}(\sim 15$ in $(3), \sim 25 \mathrm{~min}$ in $(4))$, if the SD front breaks open, corresponding to migraine with aura (MWA). (b) Statistical analysis of 8000 events plotted over MIA and TAA, see text.

we explicitly assume the presence of headache. MxWA is estimated to account for about $5 \%$ and MWA for $25 \%$ of the $30 \%$ of the subtype migraine with aura (1.2). The number of unreported cases of MxWA might be quite large, though.

Together with MWoA, three combinations exist. We predict that each migraine subform has a specific signature of SD. Characteristic for this signature is not only the spatio-temporal pattern of the course of SD in the gyrified human cortex but also the hemodynamic response and the amount of noxious substances that diffuse into the meninges. The cortical folding pattern should also influence this signature. We are currently performing a detailed analysis of this influence.

In particular, we predict that a sufficiently large surface area must be instantaneously affected and thus depleted by SD to lead into the pain phase in migraine. Therefore, we propose that if the maximal instantaneously affected area (MIA, Fig. 5b) is too small, the cascade of subsequent events causing sustained activation of trigeminal afferents is not initiated. The rational behind this is that the transmission of substances (noxious or inflammatory mediators) in the direction perpendicular to the cortical surface into the pain sensitive meninges should be significantly convergent to reach noxious threshold concentration and initiate central sensitisation of second order neurons, Fig. 6. While the flow driven by small MIA is sufficiently diluted and therefore 


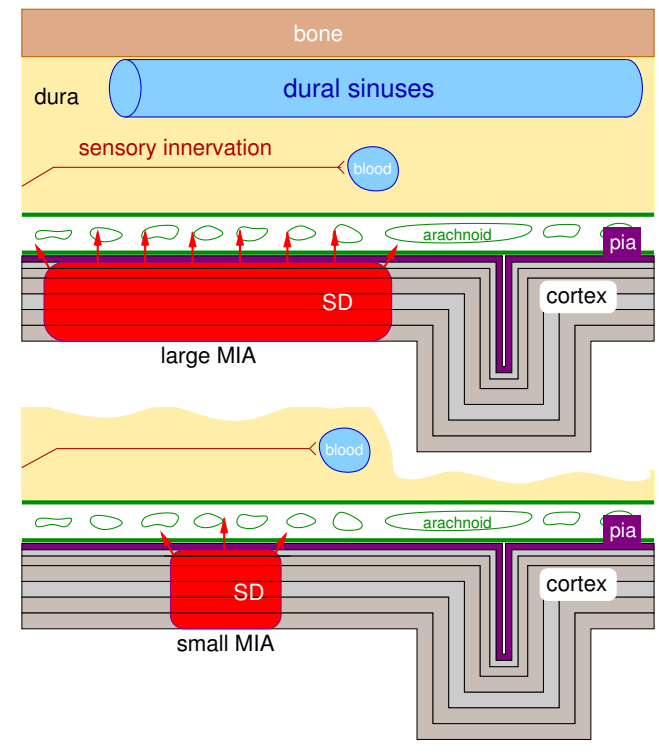

FIG. 6. (color online) Schematic representation of a cortical cross section with meninges and skull. Top: large MIA; bottom: lower MIA, not to scale; cortical thickness is at the occipital lobe about $2 \mathrm{~mm}$; diameter of MIA pain threshold is approximately $1.78 \mathrm{~cm}$.

tolerated. This makes a prediction that can be tested by noninvasive imaging.

Furthermore, only if SD assumes a spatio-temporal form that is lasting long enough $(>5 \mathrm{~min})$ and therefore also propagating farther $(>1.5 \mathrm{~cm})$, SD will cause noticeably aura symptoms. In fact, this is not a prediction. It merely reflects the diagnostic criteria of migraine aura given by the International Classification of Headache Disorders: "focal neurological symptoms that usually develop gradually over 5-20 minutes". So any neurological events that last less than $5 \mathrm{~min}$ are usually not diagnosed as migraine aura.

The pain phase is completely suppressed in cases of MxWA, if the ignition is not causing a large MIA. Such a temporal evolution in Eq. (1)-(2) is expected for initial perturbations that are just large enough to be suprathreshold stimulations and at the same time are asymmetric to lead directly into a discontinuous wave (Fig. 4D) that is slowed down. As a consequence from the model, MxWA are particularly long lasting auras, again a prediction that can be tested.

\section{G. Pain threshold determined by SD reaction-diffusion model}

The distribution of spatio-temporal SD patterns that results from local perturbation led us to suggest a pain threshold determined by MIA. This value can be estimated to be about MIA $\approx 2.5 \mathrm{~cm}^{2}$. In other words, if SD is never depleting the transmembrane gradients in cor- tical tissue over a surface area of more than $2.5 \mathrm{~cm}^{2}$ (at any given time), the pain cascade is not initiated. This corresponds to a circular area with a diameter of about $d \approx 1.78 \mathrm{~cm}$ or $0.1 \%$ of the total cortical surface. The course (2) shown in Fig. 5 has a MIA of about $4.6 \mathrm{~cm}^{2}$, while the course (3) and (4) are just above the pain threshold.

In a nondimensionalized generic model, we can only give an estimate of the surface area of the pain threshold. In fact, we rely on some self-consistency which can have biased the result as discussed briefly in the following.

To estimate surface area in units of $\mathrm{cm}^{2}$, the nondimensionalization of the SD model needs to be considered. We could have started with bistable kinetics for the activator, similar to that as suggested in the GHH model for $u=\left[K^{+}\right]_{e}$ with three roots at $3 \mathrm{mM}$ and $55 \mathrm{mM}$, for the stable fixed points, resting state and depleted state, respectively, and $10 \mathrm{mM}$ for the threshold, a value that is under physiological conditions well preserved and therefore called the ceiling level of $\left[K^{+}\right]_{e}^{43}$. Also the diffusion coefficient for potassium ions in the brain can be used. This would result in an equation structurally similar to Eq. (1) but in a dimensionalized form. If we were to add linear kinetics for an immobilized inhibitor (for the sake of argument, set in Eq. (2) $K=0$ ), there is a total of 7 parameters ( 4 for the cubic rate function of $u$, the diffusion coefficient, and 2 for the linear rate function of $v$ ). We can scale 4 quantities : the 2 concentrations of $u$ and $v$ as well as time and space. Therefore, only 3 free parameters remain in a nondimensionalized model. Since the rate function in Eq. (2) (still with $K=0$ ) is only a function of $u$ and not also $v$, this reduces further to the two parameter $\beta$ and $\varepsilon$ - for the weak limit, we consider here, this is canonical. Weak limit refers to the fact that the threshold of the local dynamics $(D=0)$ is large and the bifurcation that determines the oscillatory behavior (type I or type II) is irrelevant.

For any set $(\varepsilon, \beta)$ in the parameter plane, we can reconstruct dimensionalized space and time scales, because we know both the speed of SD and the width of the wave profile 44 . This procedure can be performed in a similar way with $K \neq 0$, although we select by the choice of the mean field feedback the size of the critical surface area. This is because the choice of $\mathrm{K}$ selects a specific path in parameter space through the saddle-node bifurcation and therefore also the "ghost" behavior, which is indirectly linked to the obtained value of the pain threshold, see Ref. ${ }^{34}$ for a detailed discussion. For the values used here, we have approximated the dimensionalized time and space scales $t_{d}$ and $x_{d}$ (and $y_{d}$ ) by $t_{d}=t / 10$ and $x_{d}=x / 8$, where $t$ and $x$ are nondimensionalized time and space scales from Eqs. (1)-(2), respectively. 


\section{DYNAMICAL DISEASE AND NEUROMODULATION}

It is often not helpful or even meaningful to talk about an upstream cause for a composite dynamical system being out of control, if the control is established in closed loop feedback between the composites. It is likely that migraine episodes are caused by events that should be described as transitions in the dynamical state of the brain. And it can take various triggers to make this transition but the cause is inherently in the dynamics. Therefore, let us address the question: Why do we want to understand migraine as a dynamical disease?

"The significance of identifying a dynamical disease is that it should be possible to develop therapeutic strategies based on our understanding of dynamics combined with manipulations of the physiological parameters back into the normal ranges" 45 . This quote provides a general answer to this question and to why therapeutic strategies that rely on the dynamics will be considered in this section.

The focus in this study is on neuromodulation techniques. One reason is because pharmacological treatment is not suitable for any direct dynamical treatment at high frequencies because most fast time scales are damped due to pharmacokinetics, that is, absorption, distribution, and metabolism, before pharmacodynamics sets in-which of course can act indirectly on fast time scales. These issues are bypassed in neuromodulation. Neuromodulation is also usually (though not always, see below) spatially confined. Since dynamical diseases are caused by transitions in both temporal rhythms and spatio-temporal patterns, neuromodulation is the natural ansatz for their treatment. "The headache future is bright for neuromodulation techniques, if we manage to understand how they work." A quote from a slide by Jan Schoenen (with permission).

Another reason is that various minimally invasive and noninvasive neuromodulation techniques are available and have been tested clinically in migraine $e^{46}$. To date, these treatment options are usually only considered in migraine in chronic cases of several disabling attacks per month that are refractory to other current treatment. But they might become available in episodic migraine, if safety issues are appropriately resolved.

\section{A. Phase-dependent mode of action}

Possible target regions are the brain structures depicted in Fig. 2. At least two distinct dynamical situations during a migraine episode should be distinguished because the optimal stimulation protocol is likely to depend not only on the mode of action but also on these different phases. The ignition phase (IP, Fig. 83) where SD is actively controlled by hyperemia, and the longer lasting acute phase (AP, Fig. $8 \mathrm{~b})$, including oligemia $(\sim 2 \mathrm{~h})$ and sustained pain activity, which can last in migraine from 4 to $72 \mathrm{~h}$.

The IP lasts about as long as SD circumscribes a large surface area in the cortical tissue, which is assumed to be above the pain threshold, Fig. 7. During the acute aura phase, there is a cortical spatio-temporal pattern of both hyperemia and oligemia related to the SD pattern. This pattern might project even to the nuclei of the MGN, that is, it is mapped in a topographic representation. The acute pain phase can overlap and last up to $72 \mathrm{~h}$. Therefore Fig. 8 oversimplifies these phases but gives a first hint on the dominant dynamics. In particular, Fig. 8 a shows the traffic that eventually results in central sensitization, while Fig. 8b shows the facilitated pain traffic during central sensitization (cf. Sec. II). As a consequence, different neuromodulation targets and/or stimulation protocols should be developed and applied for IP and AP. Various techniques are available, as briefly reviewed in the following.

\section{B. Invasive neuromodulation}

It is worthwhile to note that patients with chronic pain syndromes - but headache-free - developed headaches with migrainous features following implantation of deep brain stimulation (DBS) electrodes that target in periaqueductal grey $(\mathrm{PAG})^{47 / 48}$. This was taken as evidence for the significant role of PAG in the migraine generator (see Sec. II cf. Ref.7). Although posterior hypothalamic DBS is applied to cluster headache, DBS as an invasive method does not play a significant role in headache treatment to date. The $3 \%$ risk of bleeding is only within the upper range reported for DBS in movement disorders and other than in these disorders ( cf. Refs $^{49150}$ ), the mode

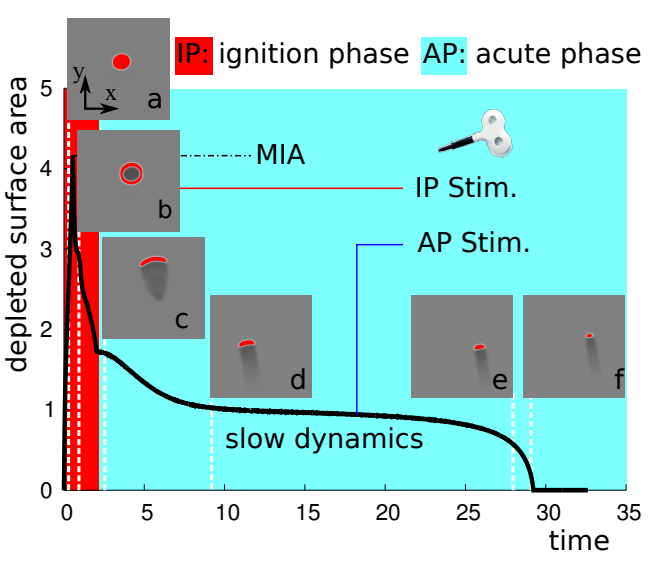

FIG. 7. (color online) Simulated course of cortical surface area depleted by during SD. Insets: snapshots of simulated SD wave in $2 \mathrm{D}$, top view, (a) ignition shortly before MIA is reached; (b) ring wave before breaking into discontineous wave; (c) discontineous wave; (d) propagation near "ghost", note the new reference frame; (e) propagation behind the ghost, new reference frame; (f) collapse. 

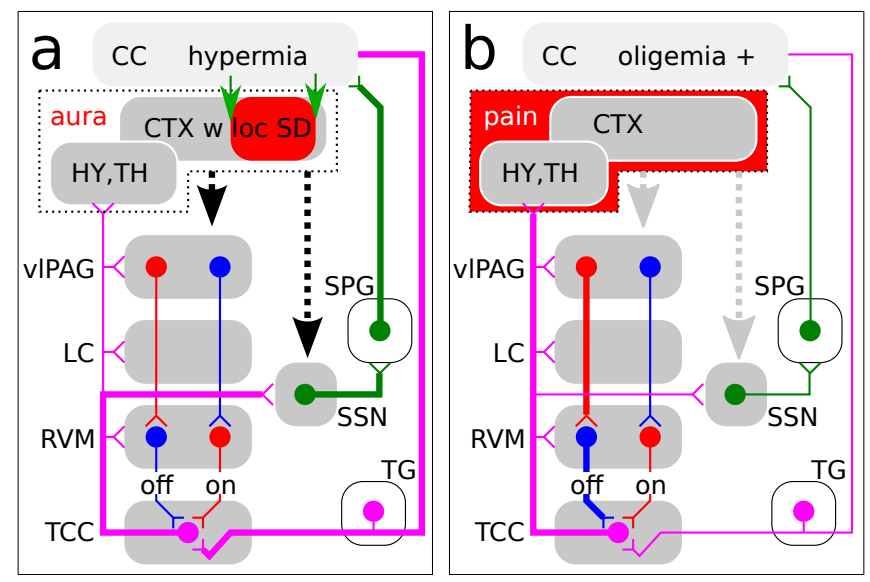

FIG. 8. (color online) Suggested facilitated flow of pain traffic in the reduced migraine generator network. (a) Flow during ignition of SD; (b) after central sensitisation.

\section{Noninvasive neuromodulation}

Transcutaneous and transcranial stimulation are the two noninvasive modes. Transcutaneous electrical nerve stimulation (TENS) modulates neural activity by targeting noninvasively peripheral nerves (cf. ONS). In migraine, a supraorbital transcutaneous stimulation (STS) was tested with a portable device that looks like a silver headband and targets the ophthalmic division of the trigeminal nerve - the first-order neuron that ends in the TCC. The stimulation was tested with biphasic rectangular $\mathrm{AC}$ impulses at $60 \mathrm{~Hz}$. The therapeutic gain $(26 \%)$ is within the range of those reported for other preventive drug and nondrug antimigraine treatments 57 . But like with ONS, the mode of action or even quantitative methods to test paradigms are currently missing, though it seems suggestive that if central sensitization of secondorder neurons are causing the pain, this method is suppressing not the sensitivity to noxious stimuli or nonnoxious stimuli but the inbound sensory traffic.

Transcranial stimulation targets the cortex. Two noninvasive neuromodulation techniques are available in migraine, transcranial magnetic stimulation (TMS) and transcranial electrical stimulation (TES). A carefully sham-controlled larger trial with a portable TMS devise was undertaken as a promising noninvasive neurostimulator for disrupting migraine attacks 58 . The simplest technology with the least safety issue is probably TES, a method that therefore might pave the road for noninvasive portable devices for episodic migraine in less disabled patients in the future. Two TES versions exist, transcranial direct current stimulation (tDCS) and alternating current stimulation (tACS). The lack of spatial specificity is, however, a critical point. Cathodal tDCS inhibits, anodal tDCS increases neuronal firing. Preliminary evidence was found for patients with chronic migraine having a positive, but delayed, response to tDCS applied to motor (anodal) and oribitofrontal (cathode) cortices 59 . Computational simulations of current flow through brain regions were used to interpret the effects. With the help of such simulations, a principal understanding of pain modulation can be gained, whether this modulation is due to the effect of TES on SD or on the dynamics of the pain matrix (in chronic pain). Furthermore, the effect of TES on deeper structures beyond the immediate cortical target regions can be estimated, including the cingulate, insula, thalamus, and brainstem 59 . This is a clinical research model that could serve as a guide to future investigations. Effective prophylactic therapy in migraine was also studied with cathodal tDCS over the visual cortex with the anode overlying the motor and sensory cortices 60 . When alternating current $(\mathrm{AC})$ is applied over the scalp it is known as tACS, but tACS has not been clinically tested in migarine. 


\section{Model-based control}

The above mentioned techniques need a model of the pathophysiological events in the target region to optimize the stimulation protocol. This is even mandatory in a closed loop feedback control, such as a Kalman filter, but it is also important for open loop control, which is the current mode in all neuromodulation for migraine. Simply said, given that we know how the hardware can interface with the wetware, what is the best software?

Quantitative models for SD are already developed in great detail. Not only macroscopic models are available, such as suggested and briefly introduced here, but also detailed models including the electrophsiological events on a cellular level, for a review see Ref.61. This opens up a model-based approach to target SD in the cortex.

Macroscopic equations of motion for SD, as Eqs. (1)(2) — which also are considered as "toy models" - have two advantages. First, they allow extensive computational simulations of spatio-temporal pattern that extend in the case of SD in migraine over several centimeters and last up to one hour. A microscopic model of SD describing the dynamics on the millisecond scale of single cells to calculate the noxious signatures that are transmitted into the meninges during one hour over several square centimeters is to crack a nut with a sledgehammer. However, the main advantage of such generic models lies in the fact that they allow insight in the phase space structure of the whole class of models they represent, Fig. 4. In this sense they are generic and well suited in particular to develop open loop control stimulation protocols.

Given the predicted spatio-temporal signatures following from the model in Eq. (1)-(2), stimulation protocols in the ignition phase and the acute phase have different control aims. The acute phase can be further subdivided into one with the aura present and one with only the headache. Furthermore, the prodromal phase exists.

The most obvious stimulation protocol can be derived for the acute phase with the aura present. In this phase the slow dynamics are governed by ghost behavior after a saddle-node bifurcation, Fig. 4d. Noise can speed up the transition time in this case and shorten the aura. Although this is not the major contribution to the pain phase as SD is below the pain threshold, once this threshold is crossed during ignition, it is likely beneficial to suppress any further release of noxious or inflammatory mediators by the decaying SD wave.

According to our prediction, the most critical phase is the ignition phase. In this phase the pain threshold will be usually crossed. It is also the shortest lasting phase of only a couple of minutes. This phase is therefore likely to be missed, if neuromodulation is not used in a closed-loop control with some sort of feedback.

Therefore, the prodromal phase becomes in particular valuable for prophylactic protocols. In this phase, cortical excitability might be temporally decreased to lower the upcoming ignition. Prophylactic protocols with cathodal transcranial direct current stimulation of the visual cortex are currently tested $\frac{60}{}$. Similar approaches should be tested with TMS, which can be more spatially selective to interact with cortical hot spots as targets.

In the acute pain phase, SD is not a target anymore. Therefore the model contributes nothing to this phase, except, as already noted above, that according to the proposed mechanism transcutaneous stimulation will in this case target the inbound sensory traffic.

\section{SUMMARY}

The migraine generator (Sec. II ) and the spreading depression (Sec. III) theory of migraine are not mutually exclusive theories of the cause of episodic migraine. Sufficient evidence speaks to the fact that both theories can be validly maintained. It may be that the migraine subtypes MWoA and MWA have different pathophysiological mechanisms, though this seems rather unlikely. Likewise, it cannot be ruled out that the phenotype of each classification subtype has different pathophysiological mechanisms.

The proposed mechanism still allows space for either of these possibilities. The unified framework, however, does not rely on either of these options. We propose that stereotype spatio-temporal signatures of SD (Fig. 5) can selectively drive rhythms in the MGN, which in turn is part of a larger network (Fig. 2) that controls the spatiotemporal dynamics of SD in the first place.

The question of the most upstream events in episodic migraine lead to a long standing controversy. To my mind, the settlement of this controversy points strongly to the need to complement clinical and experimental data with quantitative approaches, in particular computational simulations and mathematical analysis of the dynamical interaction between the migraine generator and spreading depression. This study can only outline a framework for such quantitative approaches.

\section{ACKNOWLEDGMENTS}

The author kindly acknowledges the support from the Bundesministerium für Bildung und Forschung (BMBF 01GQ1109). This research has been supported in part also by the Mathematical Biosciences Institute at the Ohio State University and the National Science Foundation under Grant No. DMS 0931642.

\section{REFERENCES}

${ }^{1}$ L.J. Stovner, K. Hagen, R. Jensen, Z. Katsarava, R.B. Lipton, A.I. Scher, T.J. Steiner, and J.A. Zwart, "The global burden of headache: a documentation of headache prevalence and disability worldwide," Cephalalgia 27, 193-210 (2007).

${ }^{2}$ J. M. Hansen, R. B. Lipton, D. W. Dodick, S. D. Silberstein, J. R. Saper, S. K. Aurora, P. J. Goadsby, and A. Charles, "Migraine 
headache is present in the aura phase: a prospective study," Neurology 79, 2044-2049 (2012).

${ }^{3}$ M.C. Mackey and J.G. Milton, "Dynamical diseases," Ann. N. Y. Acad. Sci. 504, 16-32 (1987).

${ }^{4}$ C. Weiller, A. May, V. Limmroth, M. Juptner, H. Kaube, R.V. Schayck, H.H. Coenen, and H.C. Diener, "Brain stem activation in spontaneous human migraine attacks," Nat. Med. 1, 658-660 (1995).

${ }^{5}$ KMA Welch, V. Nagesh, S.K. Aurora, and N. Gelman, "Periaqueductal gray matter dysfunction in migraine: cause or the burden of illness?." Headache: The Journal of Head and Face Pain 41, 629-637 (2001).

${ }^{6}$ Anthony W Fox, "Time-series data and the "migraine generator"," Headache 45, 920-925 (2005).

${ }^{7} \mathrm{D}$ Borsook and R Burstein, "The enigma of the dorsolateral pons as a migraine generator," Cephalalgia 32, 803-812 (2012).

${ }^{8}$ R. Burstein and M. Jakubowski, "Unitary hypothesis for multiple triggers of the pain and strain of migraine," J. Comp. Neurology 493, 9-14 (2005).

${ }^{9}$ M. M. Heinricher, I. Tavares, J. L. Leith, and B. M. Lumb, "Descending control of nociception: Specificity, recruitment and plasticity," Brain Res Rev 60, 214-225 (2009).

${ }^{10}$ GD Iannetti and A Mouraux, "From the neuromatrix to the pain matrix (and back)," Exp. Brain Res. 205, 1-12 (2010).

${ }^{11}$ M.J. Purves, "Do vasomotor nerves significantly regulate cerebral blood flow?." Circulation Research 43, 485-493 (1978).

${ }^{12}$ Joseph L Price, "Prefrontal cortical networks related to visceral function and mood," Annals of the New York Academy of Sciences 877, 383-396 (2006).

${ }^{13}$ Kevin A Keay and Richard Bandler, "Parallel circuits mediating distinct emotional coping reactions to different types of stress," Neuroscience \& Biobehavioral Reviews 25, 669-678 (2001).

${ }^{14}$ S. Akerman, P.R. Holland, and P.J. Goadsby, "Diencephalic and brainstem mechanisms in migraine," Nat. Rev. Neurosci. 12, 570-584 (2011).

${ }^{15}$ L. M. Mendell, "Computational functions of neurons and circuits signaling injury: relationship to pain behavior," Proc. Natl. Acad. Sci. U.S.A. 108 Suppl 3, 15596-15601 (2011).

${ }^{16}$ R. Burstein, M.F. Cutrer, and D. Yarnitsky, "The development of cutaneous allodynia during a migraine attack clinical evidence for the sequential recruitment of spinal and supraspinal nociceptive neurons in migraine," Brain 123, 1703-1709 (2000).

${ }^{17}$ N.F. Britton and S.M. Skevington, "A mathematical model of the gate control theory of pain," Journal of theoretical biology 137, 91-105 (1989).

${ }^{18}$ J.P. Dreier, "The role of spreading depression, spreading depolarization and spreading ischemia in neurological disease," Nat. Med. 17, 439-447 (2011).

${ }^{19}$ G. G. Somjen, "Mechanisms of spreading depression and hypoxic spreading depression-like depolarization," Physiol. Rev. 81, 1065-1096 (2001).

${ }^{20}$ J.P. Dreier, T.M. Isele, C. Reiffurth, S.A. Kirov, M.A. Dahlem, and O. Herreras, "Is spreading depolarization characterized by an abrupt, massive release of gibbs free energy from the human brain cortex?." Neuroscientist 19, 25-42 (2013).

${ }^{21}$ E. Mosekilde, J.S. Thomson, C. Knudsen, and R. Feldberg, "Phase diagrams for periodically driven Gunn-diodes," Physica D 66, 143 (1993).

${ }^{22}$ H. Bolay, U. Reuter, A.K. Dunn, Z. Huang, D.A. Boas, and M.A. Moskowitz, "Intrinsic brain activity triggers trigeminal meningeal afferents in a migraine model," Nat. Med. 8, 136-142 (2002).

${ }^{23}$ K. Eikermann-Haerter and M.A. Moskowitz, "Animal models of migraine headache and aura," Curr. Opin. Neurol. 21, 294-300 (2008).

${ }^{24}$ P. C. Tfelt-Hansen, "Permeability of dura mater: a possible link between cortical spreading depression and migraine pain? A comment," J. Headache Pain 12, 3-4 (2011).

${ }^{25}$ B. K. Ingvardsen, H. Laursen, U. B. Olsen, and A. J. Hansen, "Possible mechanism of c-fos expression in trigeminal nucleus caudalis following cortical spreading depression," Pain 72, 407415 (1997).

${ }^{26}$ M. A. Moskowitz and R. Kraig, "Comment on Ingvardsen et al., PAIN, 72 (1997) 407-415," Pain 76, 265-267 (1998).

${ }^{27}$ H. Karatas, S.E. Erdener, Y. Gursoy-Ozdemir, S. Lule, E. ErenKocak, Z. D. Sen, and T. Dalkara, "Spreading depression triggers headache by activating neuronal Panx1 channels," Science 339, 1092-1095 (2013).

${ }^{28}$ Cenk Ayata, "Cortical spreading depression triggers migraine attack: Pro," Headache: The Journal of Head and Face Pain 50 725-730 (2010), ISSN 1526-4610.

${ }^{29} \mathrm{~J}$. Olesen, B. Larsen, and M. Lauritzen, "Focal hyperemia followed by spreading oligemia and impaired activation of $\mathrm{rCBF}$ in classic migraine," Ann. Neurol. 9, 344-352 (1981).

${ }^{30}$ Martin Lauritzen, "Cortical spreading depression as a putative migraine mechanism," Trends Neurosci. 10, 8-13 (1987), ISSN $0166-2236$.

${ }^{31}$ N. Hadjikhani, M. Sanchez Del. Rio., O. Wu, D. Schwartz, D. Bakker, B. Fischl, K.K. Kwong, F.M. Cutrer, B.R. Rosen, R.B. Tootell, A.G. Sorensen, and M.A. Moskowitz, "Mechanisms of migraine aura revealed by functional MRI in human visual cortex," Proc. Natl. Acad. Sci. U.S.A. 98, 4687-4692 (2001).

${ }^{32}$ A.A.P. Leão and R.S. Morison, "Propagation of cortical spreading depression," J. Neurophysiol. 1, 33-45 (1945).

${ }^{33}$ R. P. Woods, M. Iacoboni, and J. C. Mazziotta, "Brief report: bilateral spreading cerebral hypoperfusion during spontaneous migraine headache," N. Engl. J. Med. 331, 1689-1692 (1994).

${ }^{34}$ M. A. Dahlem and T. M. Isele, "Transient localized wave patterns and their application to migraine," J. Math. Neurosci(2011).

${ }^{35}$ M. A. Dahlem and S. C. Müller, "Reaction-diffusion waves in neuronal tissue and the window of cortical excitability," Ann. Phys. 13, 442-449 (2004)

${ }^{36}$ M. A. Dahlem and N. Hadjikhani, "Migraine aura: retracting particle-like waves in weakly susceptible cortex," PLOS ONE 4 , e5007 (2009).

${ }^{37}$ M. F. James, M. I. Smith, K.H. Bockhorst, L.D. Hall, G.C. Houston, N.G. Papadakis, J.M. Smith, A.J. Williams, D. Xing, A.A. Parsons, C.L. Huang, and T.A. Carpenter, "Cortical spreading depression in the gyrencephalic feline brain studied by magnetic resonance imaging," J. Physiol. (Lond.) 519 Pt 2, 415-425 (1999).

${ }^{38}$ Nail Akhmediev and Adrian Ankiewicz, Dissipative solitons: from optics to biology and medicine, Vol. 751 (Springer, 2008).

${ }^{39} \mathrm{M}$. Vincent and N. Hadjikhani, "Migraine aura and related phenomena: beyond scotomata and scintillations," Cephalalgia 27, 1368-1377 (2007).

${ }^{40}$ M.A. Dahlem and J. Tusch, "Predicted selective increase of cortical magnification due to cortical folding," J. Math. Neurosci. 2, 14 (2012).

${ }^{41}$ M. A. Dahlem, R. Engelmann, S. Löwel, and S. C. Müller, "Does the migraine aura reflect cortical organization," Eur. J. Neurosci. 12, 767-770 (2000).

${ }^{42}$ B. Grafstein, "Neural release of potassium during spreading depression.." in Brain Function. Cortical Excitability and Steady Potentials, edited by M. A. B. Brazier (University of California Press, Berkeley, 1963) pp. 87-124.

${ }^{43}$ U. Heinemann and H. D. Lux, "Ceiling of stimulus induced rises in extracellular potassium concentration in the cerebral cortex of cat," Brain Res. 120, 231-249 (1977).

${ }^{44}$ M. A. Dahlem, F. M. Schneider, and E. Schöll, "Failure of feedback as a putative common mechanism of spreading depolarizations in migraine and stroke," Chaos 18, 026110 (2008).

${ }^{45}$ J. Belair, L. Glass, U. An Der Heiden, and J. Milton, "Dynamical disease: Identification, temporal aspects and treatment strategies of human illness," Chaos 5, 1-7 (1995).

${ }^{46} \mathrm{D}$. Magis and J. Schoenen, "Advances and challenges in neurostimulation for headaches," Lancet Neurol 11, 708-719 (2012).

${ }^{47}$ F. Veloso, K. Kumar, and C. Toth, "Headache secondary to deep brain implantation," Headache: The Journal of Head and Face Pain 38, 507-515 (2003). 
${ }^{48}$ Neil H Raskin, Yoshio Hosobuchi, and Sharon Lamb, "Headache may arise from perturbation of brain," Headache: The Journal of Head and Face Pain 27, 416-420 (2005).

${ }^{49}$ S. J. Schiff, "Towards model-based control of Parkinson's disease," Phil. Trans. R. Soc. A 368, 2269-2308 (2010).

${ }^{50}$ J. E. Rubin, C. C. McIntyre, R. S. Turner, and T. Wichmann, "Basal ganglia activity patterns in parkinsonism and computational modeling of their downstream effects," Eur. J. Neurosci. 36, 2213-2228 (2012).

${ }^{51}$ K. Paemeleire and A. M. Goodman, "Results of a patient survey for an implantable neurostimulator to treat migraine headaches," J. Headache Pain 13, 239-241 (2012).

${ }^{52}$ Stewart J. Tepper, Ali Rezai, Samer Narouze, Charles Steiner, Pouya Mohajer, and Mehdi Ansarinia, "Acute treatment of intractable migraine with sphenopalatine ganglion electrical stimulation," Headache 49, 983-989 (2009), ISSN 1526-4610.

${ }^{53}$ C.A. Popeney and K.M. Alo, "Peripheral neurostimulation for the treatment of chronic, disabling transformed migraine," Headache 43, 369-375 (2003).

${ }^{54}$ T.J. Schwedt, D.W. Dodick, J. Hentz, T.L. Trentman, and R.S. Zimmerman, "Occipital nerve stimulation for chronic headache-long-term safety and efficacy," Cephalalgia 27, 153157 (2007).

${ }^{55}$ D. Magis, M. Allena, M. Bolla, V. De Pasqua, J. M. Remacle, and J. Schoenen, "Occipital nerve stimulation for drug-resistant chronic cluster headache: a prospective pilot study," Lancet Neurol 6, 314-321 (2007).
${ }^{56}$ D. Magis, P.Y. Gerardy, J.M. Remacle, and J. Schoenen, "Sustained effectiveness of occipital nerve stimulation in drugresistant chronic cluster headache," Headache 51, 1191-1201 (2011).

57 J. Schoenen, B. Vandersmissen, S. Jeangette, L. Herroelen, M. Vandenheede, P. Gérard, and D. Magis, "Migraine prevention with a supraorbital transcutaneous stimulator. A randomized controlled trial," Neurology 80, 697-704 (2013).

${ }^{58}$ R.B. Lipton, D.W. Dodick, S.D. Silberstein, J.R. Saper, S.K. Aurora, S.H. Pearlman, R.E. Fischell, P.L. Ruppel, and P.J. Goadsby, "Single-pulse transcranial magnetic stimulation for acute treatment of migraine with aura: a randomised, doubleblind, parallel-group, sham-controlled trial," Lancet. Neurol. 9, 373-380 (2010).

${ }^{59}$ A. F. Dasilva, M. E. Mendonca, S. Zaghi, M. Lopes, M. F. Dossantos, E. L. Spierings, Z. Bajwa, A. Datta, M. Bikson, and F. Fregni, "tDCS-induced analgesia and electrical fields in painrelated neural networks in chronic migraine," Headache 52, 12831295 (2012).

${ }^{60}$ A. Antal, N. Kriener, N. Lang, K. Boros, and W. Paulus, "Cathodal transcranial direct current stimulation of the visual cortex in the prophylactic treatment of migraine," Cephalalgia 31, 820828 (2011).

${ }^{61}$ R. M. Miura, H. Huang, and J. J. Wylie, "Cortical spreading depression: An enigma," Eur. Phys. J. Spec. Top. 147, 287-302 (2007).

${ }^{62} \mathrm{~A}$. May, "A review of diagnostic and functional imaging in headache," J Headache Pain 7, 174-184 (2006).

${ }^{63}$ D. Pietrobon and J. Striessnig, "Neurobiology of migraine," Nat. Rev. Neurosci. 4, 386-398 (2003). 